\title{
PARTICLE VELOCITY MEASUREMENT IN SWIRL FLOW, LABORATORY STUDIES
}

\author{
${ }^{1}$ Hari Prasad Neopane*, ${ }^{1}$ Bhola Thapa, ${ }^{2}$ Ole Gunnar Dahlhaug \\ ${ }^{1}$ Department of Mechanical Engineering, Kathmandu University (KU), Nepal \\ ${ }^{2}$ Waterpower Laboratory, Norwegian University of Science \& Technology (NTNU), Norway \\ *Corresponding author: hari@ku.edu.np \\ Received 28 November, 2011; Revised 14 January, 2012
}

\begin{abstract}
This paper presents the laboratory studies of particle velocity measurement in highly swirl conditions similar to turbine flow in curved path. It includes a brief description of the developed test rig, concept of critical diameter of particle inside a Francis turbine and experimental analysis. When a particle is flowing in swirl flow, drag force and centrifugal force are two major forces influencing the particle equilibrium. The equilibrium of these two forces provides a critical diameter of the particle. While, a particle larger than the critical diameter move away from the centre and hit the wall, a particle smaller than the critical diameter flows along with the water, and ultimately sinks. For critical diameter, the particle continues to rotate in the turbine. Different shapes and sizes of particles were tested with the same operating conditions and found that triangularly shaped particles were more likely to hit the suction side of the guide vane cascade. Furthermore, this study supports the concept of separation of particles from streamlines inside the test rig, which led to the development of an operating strategy for a Francis turbine processing sediment-laden water. This study also permitted experimental verification of the size and the shape of a particle as it orbits in the turbine, until either the velocity components are changed or the particle became smaller.
\end{abstract}

Keywords: Swirl flow, particle velocity, critical diameter, drag co-efficient

\section{INTRODUCTION}

Swirl flow can be used as a mechanism for separation of particles from the fluids creating a centrifugal force as in many separation processes [3]. Solid particles of different shapes and sizes play a significant role in many separation processes $[1,9,10,11,14]$. The separation of particles of different shapes and sizes depends upon the variations in behavior of the particles when subjected to the action of moving fluid. A particle falling in an infinite fluid under the influence of gravity will accelerate until the resistance force that includes buoyancy and drag exactly balances the gravitational force. The constant velocity reached at that stage is called the terminal velocity. The resistive drag force depends upon an experimentally determined drag coefficient. The separation of particles from streamline depends upon acceleration of particles, which further depends on profile and curvatures of runner blade and bucket. Together with several parameters affecting erosion rate, the modification in design in term of turbine size, profile and curvatures also play a vital role for reducing erosion rate $[1,17]$. However, this aspect is not explored at large extent.

Few studies have been done in the field of particle transport and separation in erosion test rig together with cyclone separator and conveying of particles in process industry. Chevallier and Vannes [2] carried out numerical and experimental study of interaction between particle and specimen. They studied the particle speed in non-uniform flow by generalizing Basset, Boussinesq and Oseen's expression that gives equilibrium of the particle. The added weight effect, effect of static and viscous pressure, Archimedes thrust and gravity force are 
neglected. When the ratio $\rho / \rho_{p}$ is low $\left(<10^{-3}\right)$, and if the particle and fluid acceleration are of the same order, those effects become negligible. After all these simplifications, the equation of motion of particles becomes,

$$
\frac{\pi \cdot d^{3}}{6} \cdot \rho_{p} \cdot \frac{d V}{d t}=\frac{\pi}{8} \cdot d_{p}^{2} \cdot \rho \cdot C_{D} \cdot(V-C) \cdot\left|V_{p}-C\right|
$$

Equation 1

Here, $\mathrm{dp}$ is the diameter of the particle, $\rho_{p}$ is the density of the particle, $\rho$ is density of the fluid. The fluid velocity is $C$, and particle velocity is $V$. The coefficient of $\operatorname{drag}\left(C_{D}\right)$ is;

$C_{D}=\frac{24}{R_{e, p}} \cdot\left(1+0.15 \cdot R_{e, p}^{0.687}\right)$

Equation 2

Similarly, Tabakoff et al. [15], used simplified governing equation of force of interaction of particle motion in the turbo-machinery flow with reference to cylindrical coordinates relative to frame of reference, fixed with respect to the rotating blades as shown below,

$$
\bar{F}=\frac{3}{4} \cdot \frac{\rho}{\rho_{p}} \cdot \frac{C_{D}}{d} \cdot\left[\left(V_{r}-\frac{d r_{p}}{d t}\right)^{2}+\left(V_{\theta}-\frac{d\left(r_{p} \cdot \theta_{p}\right)}{d t}\right)^{2}+\left(V_{z}-\frac{d z_{p}}{d t}\right)^{2}\right]^{\frac{1}{2}} \cdot\left(\bar{V}-\overline{V_{p}}\right)_{\text {Equation } 3}
$$

Here, $\mathrm{rp}, \theta_{p}$ and $\mathrm{zp}$ define the particle location in cylindrical coordinates. Similarly, $\mathrm{Vr}, v_{\theta}$ and $\mathrm{Vz}$ represent relative gas velocities in the radial, the circumferential and the axial directions respectively. This equation includes centrifugal force as well as Coriolis force. The forces due to gravity and inter particle interaction are negligible in the case of turbo machinery. The drag coefficient $\left(\mathrm{C}_{\mathrm{D}}\right)$ is a function of particle Reynolds number. Martin Rhodes, [8] considered, $C_{D}=0.44$ for large $R_{e, p}(>500)$, and provided equation 4 for $C_{D}$ for $\mathrm{R}_{\mathrm{e}, \mathrm{p}}$ range, 0.2 - 500 .

$$
C_{D}=24 \cdot\left(R_{e, p}^{-1}+0.167 \cdot R_{e, p}^{-\frac{1}{3}}\right)
$$

Equation 4

\section{Characterization of non spherical particles}

Particles in water may range in size from a few nanometers to few millimeters in dimension. Natural particles also have various shapes, including rod, plate, and sphere, with many variations in between, which make the treatment of the particle size difficult. The discussion is vastly simplified if the particles are considered spherical. In this case only one size parameter is needed (the diameter) and hydrodynamic properties are much more easily treated. Of course, non-spherical particles also occur in natural water, and some way of characterizing them is essential. A common concept is that of the 'equivalent sphere' based on a chosen property of the particles [5]. For instance, an irregular particle has a certain surface area and the equivalent sphere could be chosen with the same surface area. The 
surface area of sphere with diameter $\mathrm{d}$ is just $\pi \cdot d^{2}$ [8]. So, if the surface area of the nonspherical particle is known, the equivalent spherical diameter can easily be calculated. For an object of a given volume, the sphere has minimum surface area and the volume (or mass) of a given particle must be equal to or less than that of the sphere. Another common definition of equivalent spherical diameter is based on sedimentation velocity. In this case, from the sedimentation velocity and density of particle, the diameter of a sphere of the same material that would settle at the same rate can be calculated. This is sometimes called the 'Stokes equivalent diameter'.

\section{OBJECTIVE OF EXPERIMENT}

The objectives of development of experimental set-up and conducting experiment are as follows:

- To study the particle separation process in the flow in the curved path

- To study the forces on the particle on rotational motion or swirl

- To investigate the velocity and the drag coefficient relations based upon different shape and size of the particle in swirl flow

- To establish an operating strategy for Francis turbine operating on sediment- laden water

\section{Description of test rig and test procedure}

A test rig was developed and designed at the waterpower laboratory, Norwegian University of Science \& Technology, Norway, to create strong swirl flow, similar to the flow between the guide vane outlet and the runner inlet of a turbine. There was a provision to introduce particles, with sizes ranging from 1 to $10 \mathrm{~mm}$, into the swirl, and to observe the motion of the particles from Plexiglas windows located on the cover of the tank using a high-speed digital camera.
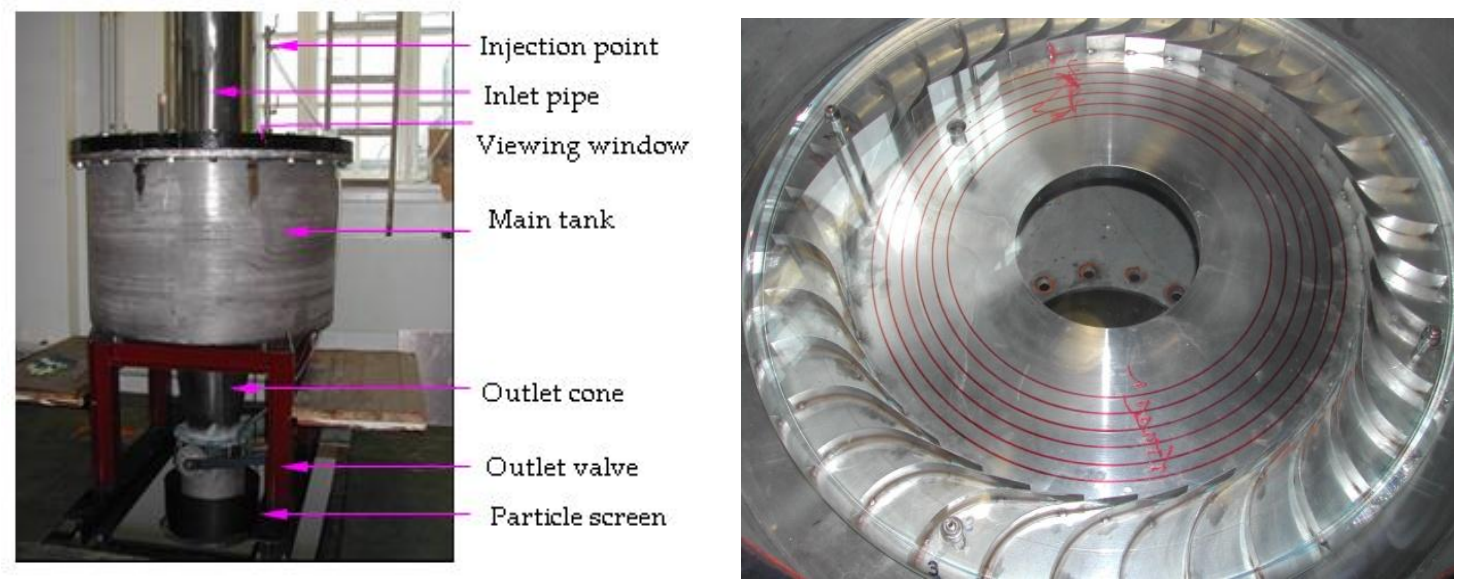

Figure 1 (a) Outside views

Figure 1 (b) Middle plate with vanes and Plexiglas 
The experimental set up, as shown in Figure 1, consisted of a main tank (1100 mm diameter and $700 \mathrm{~mm}$ height), $400 \mathrm{~mm}$ diameter inlet pipe and outlet cone with valve. The main tank and other components of the test rig were designed and dimensioned for $50 \mathrm{~m}$ of head in order to carry out the experiment in high velocity. The main tank consisted of two compartments with a $250 \mathrm{~mm}$ diameter opening at the centre of the plate. This plate divides the main tank into two compartments. Thirty-six curved vanes, which resemble guide vanes of a Francis turbine, with a radius of $100 \mathrm{~mm}$ toward the inlet and a $90 \mathrm{~mm}$ straight section toward the outlet, were fixed at a pitch circle diameter of $900 \mathrm{~mm}$ to the middle plate in such a way that the inlet velocity direction should be almost in the radial direction and the outlet would be $10^{0}$ to the tangent. Each vane cascade has $30 \mathrm{~mm}$ x $5 \mathrm{~mm}$ opening and altogether 36 numbers of vanes provide total opening area is about $5.4 \times 10^{-6} \mathrm{~m}^{2}$. These vanes were located in between the upper part of the middle plate and the bottom part of a $50 \mathrm{~mm}$ thick, $950 \mathrm{~mm}$ diameter transparent Plexiglas plate. Peripheral component $\left(\mathrm{C}_{\mathrm{u}}\right)$ of absolute velocity can be obtained from the orientation of blade angle, which is fixed at $10^{0}$ in this experimental set-up. However this angle varies at different operating conditions in actual hydropower plant. The peripheral component is caused to create a centrifugal force of particle whereas the radial component is caused to create a drag force of particle. From this arrangement, the vanes caused swirl inside the tank, which further simulate the swirl flow created by guide vanes in a Francis turbine. The swirl flow in between the Plexiglas and the middle plate could be clearly observed from the top of the Plexiglas windows, which satisfied a free vortex relation towards vanes whereas, force vortex flow exist towards the centre of the tank. The edge of the Plexiglas was made uniform to ensure uniform flow.

The top cover of the tank was fitted with five transparent windows of Plexiglas. Four of those transparent windows of $160 \mathrm{~mm}$ diameter were located at $90^{0}, 180^{0}, 270^{0}$ and $360^{0}$ respectively at the pitch circle diameter $700 \mathrm{~mm}$ to observe the motion of the particles, and the fifth window of $275 \mathrm{~mm}$ diameter was located at $315^{\circ}$ at $700 \mathrm{~mm}$ pitch circle diameter to measure the velocity of the flow (after inserting a Pitot tube as shown in Figure 3) and to observe the velocity of particle. The particle injection point was located at $279 \mathrm{~mm}$ from the centre of the tank. The particle was released at the bottom of the Plexiglas through a $15 \mathrm{~mm}$ diameter pipe and valves arrangement as shown in exploded view in Figure 2. Particles up to $10 \mathrm{~mm}$ in diameter were tested in this experiment.

To release particle, first lower valve close and inserted particle from the upper valve. Then upper valve close and lower valve open. Once lower valve opened, the particle sinks and drops in between Plexiglas and steel plate. Depending upon the velocity at the point of the injection, the particle either moves towards outer radius or follow flow direction heading towards outlet at the centre of the tank and ultimately sinks.

A manometer was fitted into the injection pipe and valves arrangement in order to measure the inlet pressure of the tank in the swirl flow field. There were two additional manometers located in the system. One was connected to an air bleeding valve located at $390 \mathrm{~mm}$ from the centre of the tank for measuring the inlet pressure, and another was fitted at the outlet of cone for measuring the pressure at the outlet. The flow rate was calculated with the help of an ultrasonic flow meter fitted in the inlet pipe. 


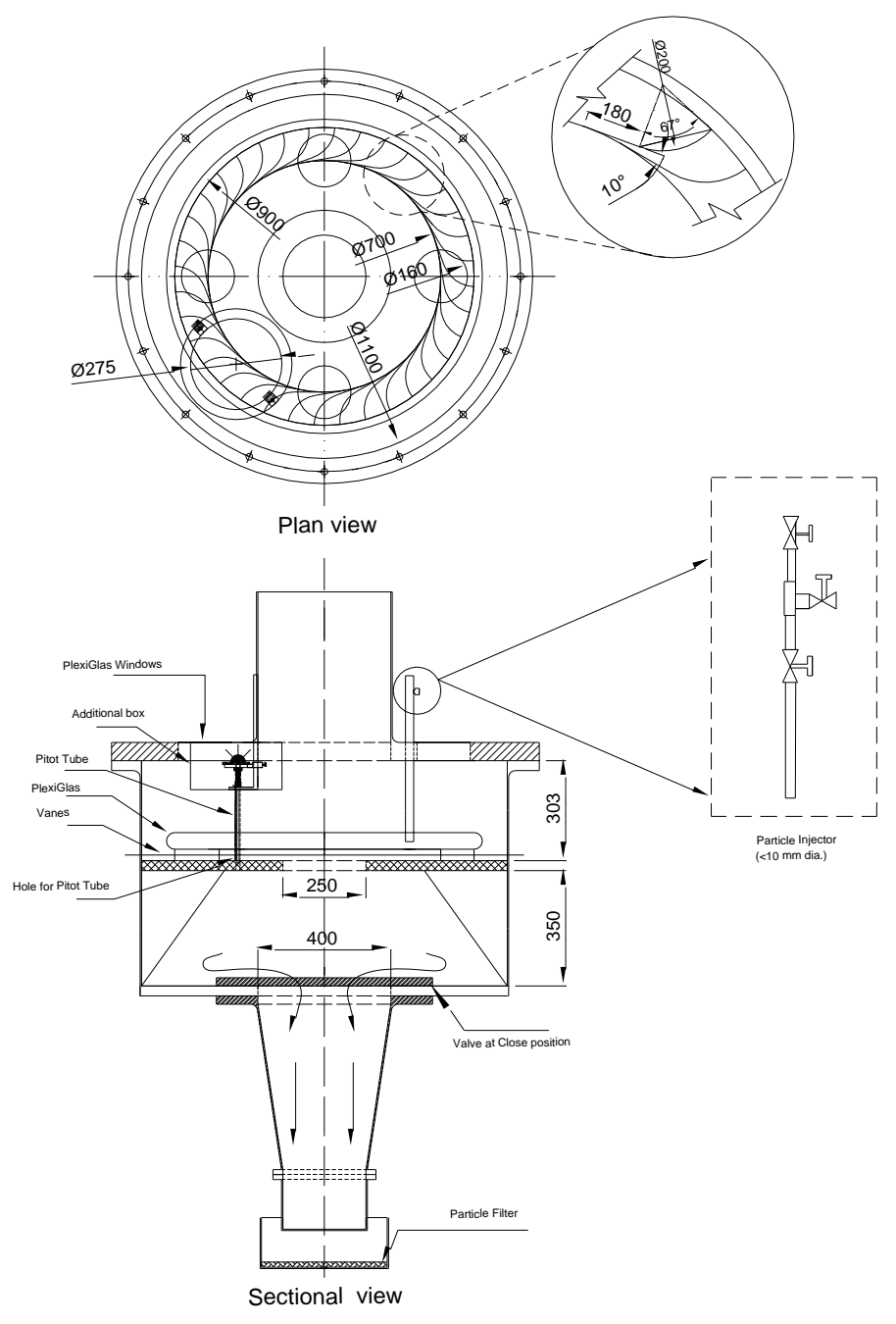

Figure 2 Schematic diagram of experimental set up

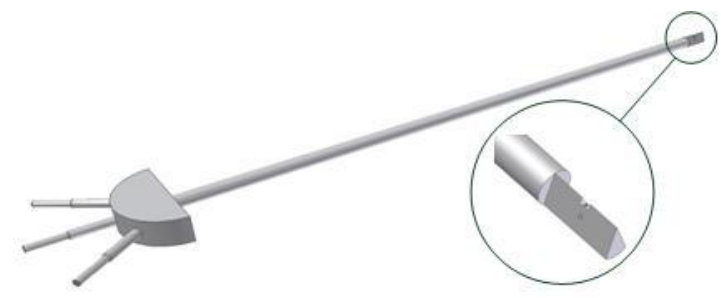

Figure 3 Pitot tube

The main purpose of this experiment was to determine the velocity of the particle that would flow along with water in the given flow condition. To achieve this, firstly, the operating condition was set at a certain velocity level by controlling the valve opening. Then the particle was injected and the motion of the particle was observed through the Plexiglas windows with the help of the high-speed camera. The particle image was saved and analysis was performed based upon the time required to move the particle from one fixed location to 
another fixed location. Location of the particle was identified based upon the radial and angular position of particle inside the test rig. In order to calculate the correct location of the particle inside the test rig, radial and angular markings were inscribed on the middle plate at diameters of $219 \mathrm{~mm}, 239 \mathrm{~mm}, 259 \mathrm{~mm}, 279 \mathrm{~mm}, 299 \mathrm{~mm}, 309 \mathrm{~mm}$ and 22.5 degrees interval respectively. Using these markings, six different circles and sixteen different angular lines could be clearly observed in the middle plate.

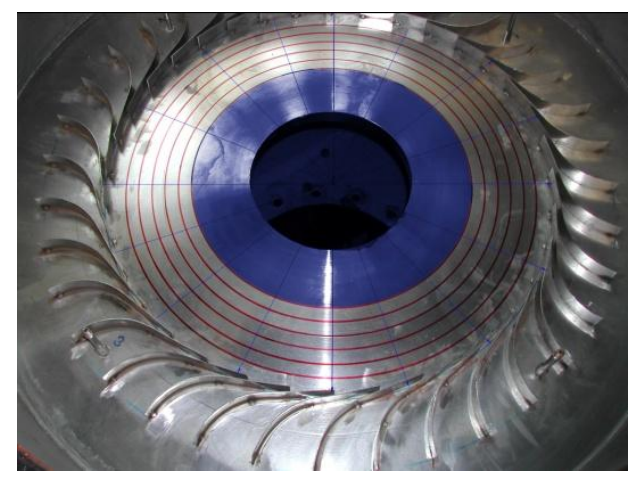

Figure 4 Photograph of middle plate with radial and angular markings

\section{Visualization of particle motion}

In order to find out the size of the particle which will flow along with water in a given flow condition, and to verify the validation of critical diameter expression that derived in equation 7, six different particles having different densities were considered. The experiments were done based upon different shape and size of aluminium, brass, ceramic, plastic, sand and steel particles. Most of these particles rotating at low velocity can be clearly seen by naked eye. However, as the velocity of particle increase it was not possible to visualize the location of particles in flow field by naked eye but the location of small particles at higher velocity was visualized by high speed video camera. The high speed digital camera was used to compare the rotation radius for different shape and size of the particles at different operating conditions. The equilibrium condition was clearly observed with adjustment of flow and sizes of the particles for most of the samples except with plastic particles. Since the density of plastic particle is less than the density of water, most of these particles were floated with water. At equilibrium condition, the rotation radius of particle was observed very close to the injection radius of particle at $279 \mathrm{~mm}$ from the centre of the tank. The larger size of the particles that was assumed larger than critical diameter of particle on that particular operating condition was clearly observed by hitting the outer wall or suction side of the guide vane cascades in this experimental set up and the smaller size of the particle was observed clearly by flowing along with water and ultimately sinks.

\section{Particle in Swirl flow}

When particles travel in swirling flow toward a turbine's outlet, which is located in the centre as shown in Figure 5, the particles will be exposed to two main forces. Centrifugal force $\left(F_{c}\right)$ moves the particles away from the centre, while the drag force $\left(F_{D}\right)$ pulls them toward the outlet, which is in the centre, or toward the runner in the case of a Francis turbine [16]. These two forces are given by the following equations $[11,16]$. 


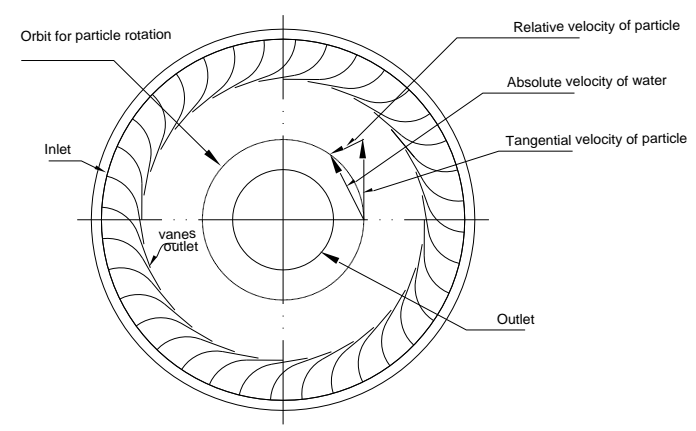

Figure 5 Illustration of particle flow in spiral swirl

$$
\begin{aligned}
& F_{c}=\rho_{p} \cdot \frac{\pi \cdot d^{3}}{6} \cdot r \cdot \omega^{2}=\rho_{p} \cdot \frac{\pi \cdot d^{3}}{6} \cdot \frac{C_{u}{ }^{2}}{r} \\
& F_{D}=\frac{1}{2} \cdot C_{D} \cdot \rho \cdot C_{m}{ }^{2} \cdot A_{p}
\end{aligned}
$$

Equation

Following three conditions prevail in such case:

- Particle will stay at the orbit of radius $\mathrm{r}$, if $F_{c}=F_{D}$

- Particle will strike outer wall, if $F_{c}>F_{D}$

- Particle will flow along with water towards the centre of the tank, if $F_{c}<F_{D}$

At equilibrium, these two forces balance each other and a particle of a given diameter will stay at an orbit of radius $(r)$ until either the velocity component is changed or particles become smaller by fracture due to impact. The diameter of a particle $(d)$ for the equilibrium condition is given by equation 7 . This is called the critical diameter $[11,14]$.

$d_{c}=\frac{3}{4} \cdot C_{D} \cdot\left(\frac{\rho}{\rho_{p}}\right) \cdot\left(\frac{C_{m}}{C_{u}}\right)^{2} \cdot r$

Equation 7

The drag force is caused by the relative velocity of particles in radial direction (i.e. towards the centre of the tank), and centrifugal force is caused by the velocity of particle in tangential direction (i.e. away from the centre of the tank).

The phenomenon of particle motion discussed here is similar to swirl flow in between guide vane outlet and runner inlet of reaction turbine. Once the larger particles enter in to the swirl flow it will be rotating and continuously hitting the suction side of guide vanes. Direct application of this concept is applied as an operating strategy for Francis turbines operating 
in sediment laden water. Guide vane position can be manipulated to maintain a velocity ratio in such a way that the particle of given size should flow along with water.

A damage caused by the larger particles has been observed in Tokke hydropower plant in Norway. The damage of suction sides of guide vanes is clearly observed as shown in Figure 6.
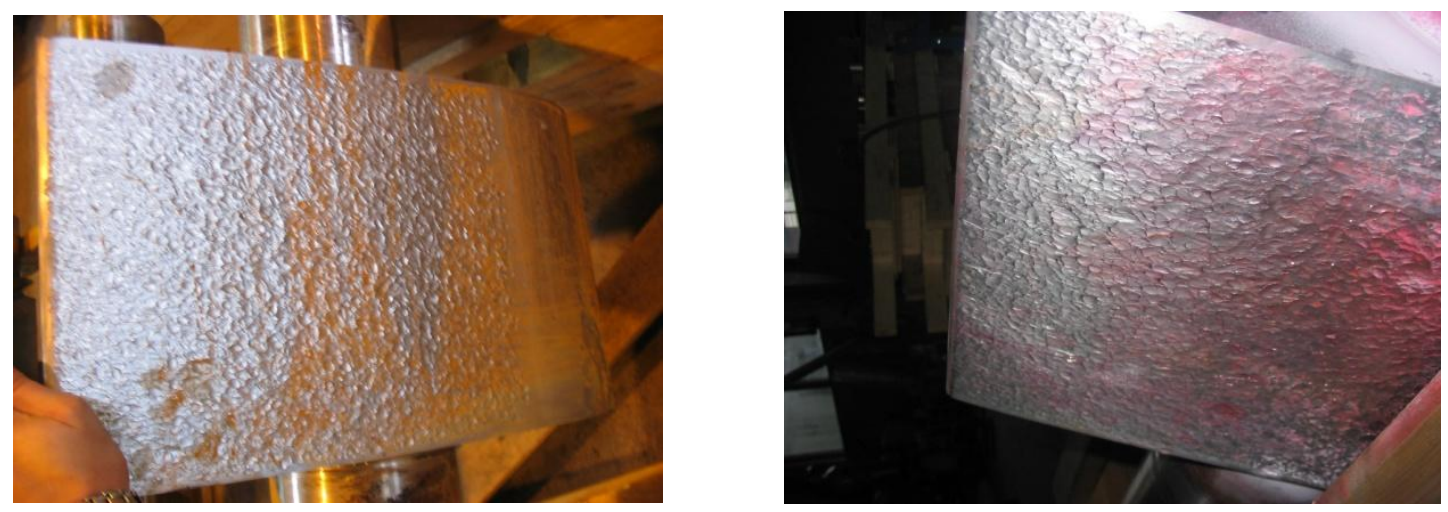

Figure 6 Erosion damage of suction side of Tokke Guide Vane by large particles

The particles of higher size are settled down or removed before it enters turbine. The unsettled large size particles, especially in the monsoon season pass thorough turbines. The guide vanes of Francis turbine can have maximum angle up to as low as $12^{\circ}$ at full load. Whereas this angle could go up to $40^{\circ}$ in some of the turbine design. The guide vane angles for some of Norwegian power plants are presented in Table 1. This inclination of guide vanes creates swirl flow in Francis turbine and the magnitude of swirl depends upon angle of inclination of guide vanes. Even though irregular shape of sand particles could have higher drag coefficient compared to spherical one, the observation from the test rig can be utilized for determining the size of particle which will remain rotating in the swirl flow in between guide vane outlet and runner inlet. Any particles higher than this size continuously hit the guide vane outlet region at suction side and damage them severely.

The diameter obtained from equation 7 can be considered as critical diameter. All the particles larger than critical diameter will remain rotating in the swirl flow hitting the guide vane wall. On the other hand all the particles smaller than critical size flows through turbines. The relation between critical diameter and runner inlet diameter is shown in Figure 7- a. This relation is plotted based upon drag coefficient 0.1 . At higher Reynolds number $\left(R_{e}>10^{6}\right)$, the drag coefficient is around 0.1 to 0.2 . Similarly, the relation between particle size and drag coefficient for turbine of radius $1 \mathrm{~m}$ at inlet is shown in Figure 7-b. This figure indicates that the sand particles of diameter higher than $0.9 \mathrm{~mm}$ will stay rotating in the swirl flow and damage guide vanes positioned around $10^{\circ}$. This figure also shows that smaller turbines are more prone to sand erosion because smaller turbines are having small critical diameter, for example particles as small as $0.7 \mathrm{~mm}$ may remain rotating in the turbines of inlet diameter $800 \mathrm{~mm}$. 
Table 1: The guide vane maximum angle at full load condition, Source: NTNU [11]

\begin{tabular}{|l|l|l|l|}
\hline S.N. & Power plant & Specific Speed $\left(\mathrm{n}_{\mathrm{s}}\right)$ & Maximum Guide vane \\
angle at full load $\left(\mathrm{a}_{0}\right)$
\end{tabular}

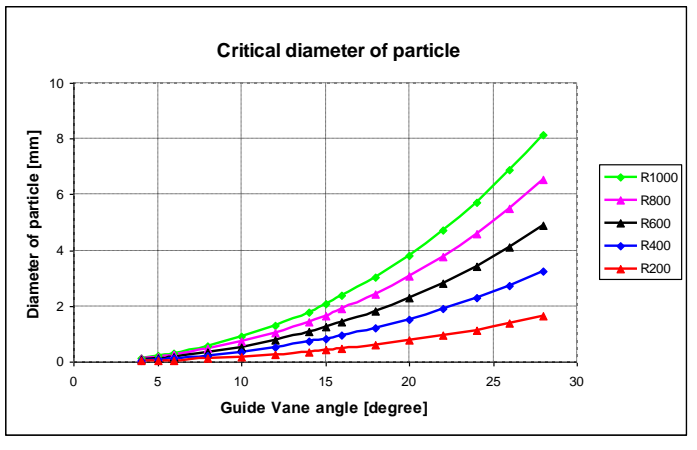

(a) Size of the runner

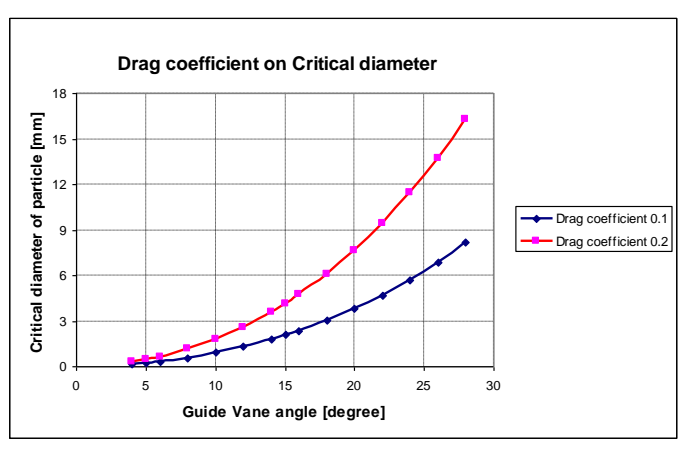

(b) Drag co-efficient

Figure 7 Critical diameter relation based upon size of the runner and drag co-efficient 
This result can be utilized as guidelines for operating strategy for Francis turbine operating in sand laden- water. If the particle size flowing with water is larger than critical sizes of the particle, the turbine should not be operated at low guide vane opening.

\section{RESULTS AND DISCUSSION}

Velocities of particles of different sizes and shapes are shown in Figures 8-10. The velocity of the particle will increase if the operating head increases. The size of the particle is inversely proportional to the velocity of the particle, and it also depends upon the shape of the particle. Different shapes of particles were tested, and it was determined that sphericallyshaped particles had higher settling velocities than particles with other shapes.

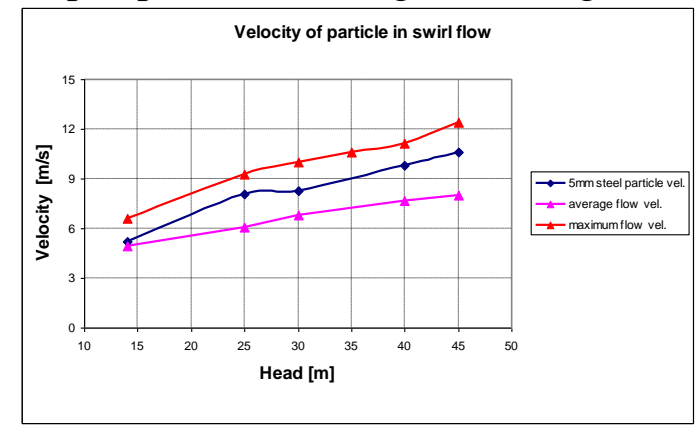

(a) Head

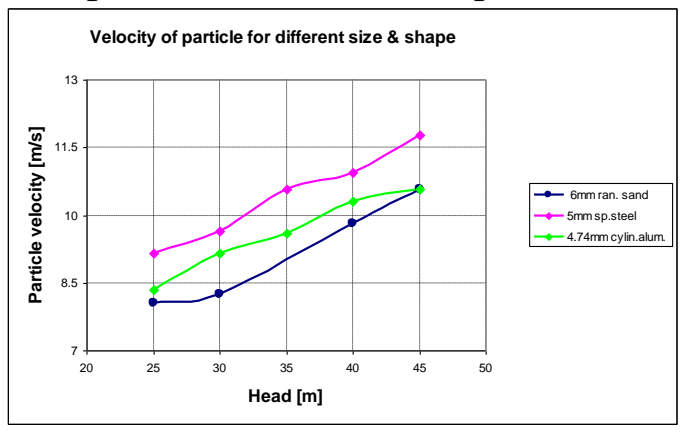

(b) Size and Shape

Figure 8 Particle velocity and head relation for different size and shape of particle

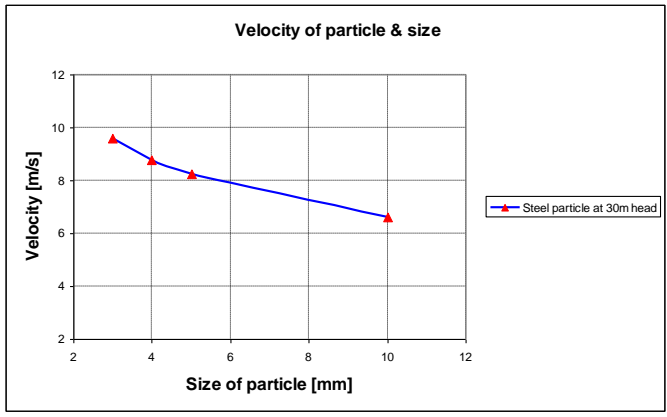

(a) Different size

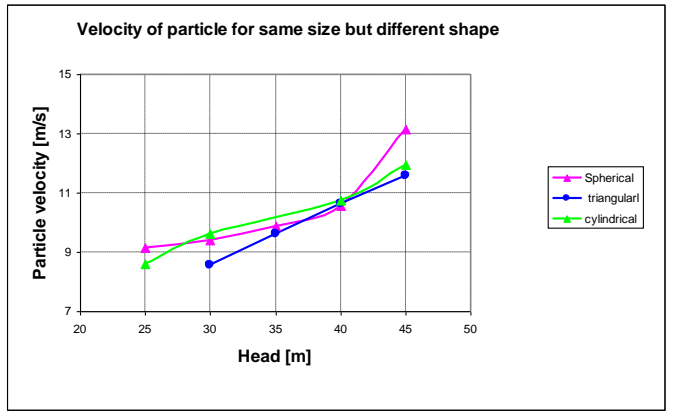

(b) Same size but different shape

Figure 9 Particle velocity for different size and same size with different shape

Many natural particles are usually in non spherical shape. These particles will tend to have lower settling velocities because both decreases in spheroid and increases in angularity tend to decrease velocities. Furthermore, larger cross-sectional areas tend to be directed perpendicular to the transport path. As a result, higher co-efficient of drag, higher rotational motion and more separation of flow likely to occur and hence more erosion rate is anticipated. 


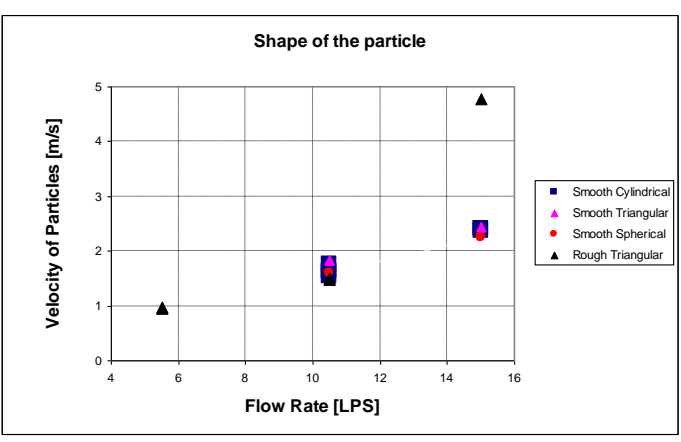

(a) Different particle with different shape

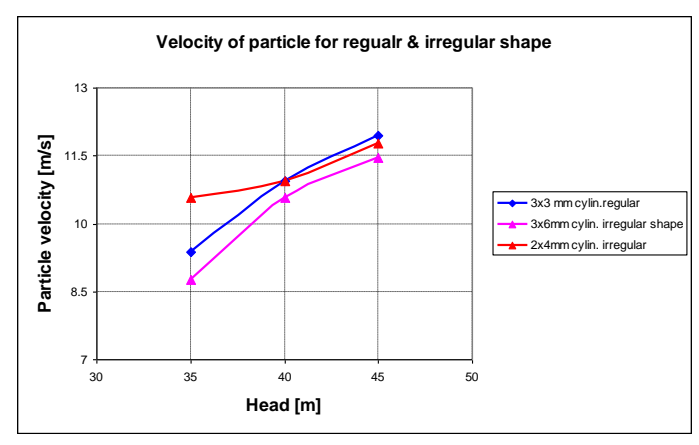

(b) same particle with different shape

Figure 10 Particle velocity relations for same/different particle with different shape

If centrifugal force and drag force on the particle are equal, the particle will rotate exactly at the injecting radius, but if those forces do not balance each other, there will two possibilities. The particle either moves toward the inner radius and ultimately sinks or moves toward the outer radius and ultimately hits the sides of the vanes. The equilibrium condition was observed for a given particle after manipulating the flow velocity, providing verification that the different forces were balanced in the test rig. This also revealed that the particle with a given diameter would stay at the orbit of the injecting radius until either the velocity components were changed or the particle became smaller by fracturing due to impact with the outer wall. Different shapes of particles were tested with the same operating conditions as shown in Figure 11, and it was determined that triangularly-shaped particles were more likely to hit the suction side of the guide vane cascade. The radius at which the particle is moving, called rotation radius. This rotation radius also varied with different shapes of particles, which further supports the influence of shape factor for particle velocity calculation.

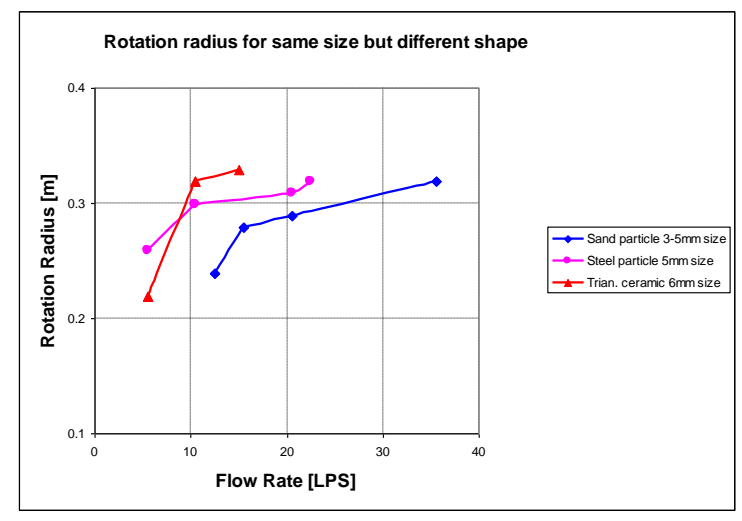

Figure 11 Rotation radius for particles of the same size but different shapes 
Drag co-efficient was also calculated and plotted versus different shapes, sizes and particle Reynolds number as shown in Figures 12 and 13.

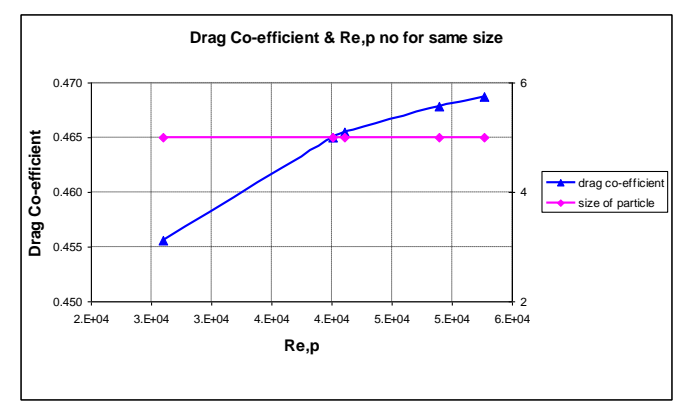

(a) Same size

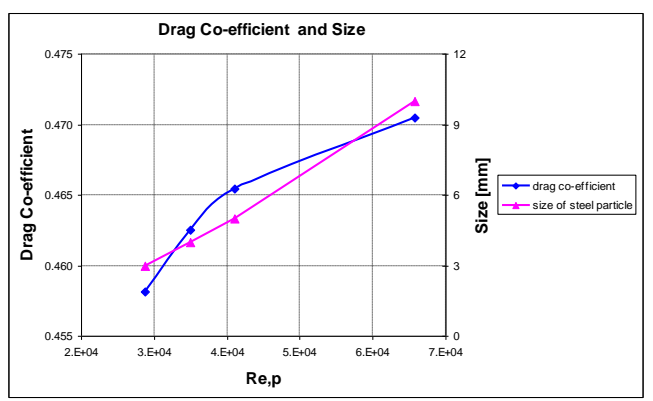

(b) Different size

Figure 12 Drag co- efficient relation for same/different size of particle

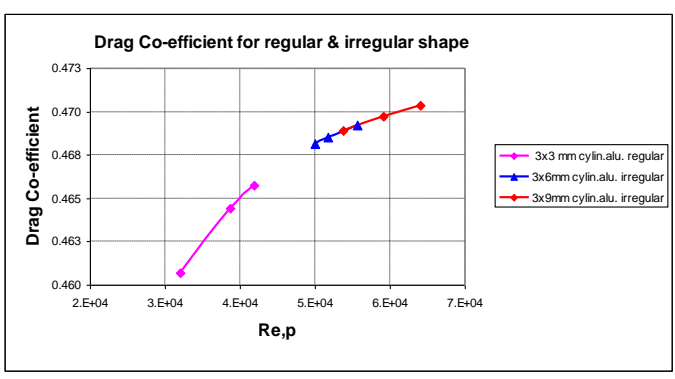

(a) Same particle different shape

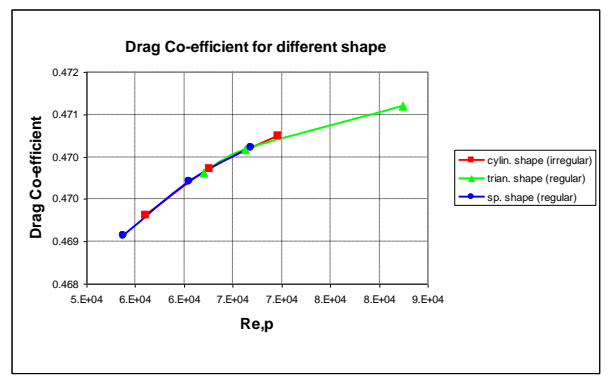

(b) Different particle different shape

Figure 13 Drag co-efficient relation for regular and irregular shape of same/different particle

The drag co-efficient, $C_{D}$ is a non-dimensional number that depends on the shape of the particle, the fluid kinematic viscosity and grain size. It has been found that the effect of the shape of non-spherical particles on their drag co-efficient can be defined in terms of its spheroid. Moreover, shape affects drag co-efficient far more in the Intermediate and Newton's law regions than in the Stokes' law region. However, the variation of drag coefficient in the Newton's law region is not so significant, but the influence of size and shape of the particle has been clearly demonstrated. The result shows that the triangularly-shaped particles have a higher drag co-efficient than other shapes because the angular particles also tend to have lower settling velocity than the spherical ones.

\section{CONCLUSIONS}

The size of a particle is inversely proportional to the velocity of the particle, and it was determined that spherically shaped particles had higher settling velocities than particles with other shapes. However, non-spherical shape of the particles will tend to have lower settling velocities because both decreases in spheroid and increases in angularity tend to decrease velocities. Moreover, larger cross-sectional areas tend to be directed perpendicular to the 
transport path. As a result, higher co-efficient of drag, higher rotational motion and more separation of flow are likely to occur and hence more erosion rate was predicted. The roles played by the shape of the particle significantly affect erosion rate prediction inside the Francis turbine components.

Furthermore, it has been found that the erosion process is strongly dependent on the particle size, shape, concentration, and operating conditions of the turbine. The reduction of the erosion is not only linked to the reduction of particle velocity but also is linked to the reduction of separation of flow, which further depends on shape, size, and concentration of the particle. The significant reduction of erosion rate can be achieved by operating turbine at best efficiency point. The full load operation reduced efficiency, increased turbulence, and increased relative velocity of flow at outlet of the blades.

\section{REFERENCES}

[1] Brekke Hermod, Design of hydraulic machinery working in sand laden water, In: Duan C G \& Karelin VY (eds), Abrasive erosion and corrosion of hydraulic machinery, pp 155-181, Imperial college press, London, 2002.

[2] Chevallier P \& A B Vannes, Effects on a sheet surface of an erosive particle jet upon impact, Wear 184(1) (1995) 87.

[3] Doby M J, A F Nowakowski, E.Nowakowski, E. Nowak, and T. Dyankowski, Numerical and experimental examination of swirl flow in a cylindrical container with rotating lid, Minerals Engineering, 20(4) (2007)361.

[4] Gabitto J \& C Tsouris, Drag co-efficient and settling velocity for particles of cylindrical shape, Powder Technology, 183(2) (2008) 314.

[5] Gregory J, Particles in water properties and processes, University College London England, CRC press, Taylor \& Francis Group, (2006)9.

[6] Haider A \& O Levenspiel, Drag coefficient and terminal velocity of spherical and non-spherical particles, Powder Technology, 58(1) (1989) 63.

[7] K Harris, Sediment transport processes in coastal environments, Lecturer notes, January 21, 2003.

[8] Martin Rhodes, Introduction to Particle Technology, Second edition, (2008) 29.

[9] Neopane H P, Ole G Dahlhaug \& B Thapa, Alternative Design of a Francis Turbine for Sand Laden Water. Proc. International Conference on Small Hydropower - Hydro Sri Lanka, 2007.

[10] Neopane H P, Ole G Dahlhaug \& B Thapa, Experimental examination of the effect of particle size and shape in hydraulic turbines, Waterpower XVI, Spokane, Washington, USA, 2009.

[11] Neopane H P, Sediment erosion in hydro turbines, $\mathrm{PhD}$ thesis, Norwegian University of Science and Technology (NTNU) Trondheim, Norway, Faculty of Engineering Science and Technology, Department of energy and process engineering, 2010. 
[12] Sheldon G L \& A Kanhere. An investigation of impingement erosion using single particles, Wear, 21(1) ( 1972) 195.

[13] Truscott G F, A literature survey on abrasive wear in hydraulic machinery, Wear, 20(1) (1972) 29.

[14] Tabakoff W, A. Hamed, et al., Investigation of gas particle flows in an erosion wind tunnel, Wear, 86(1) (1983) 73.

[15] Tabakoff W, High-temperature erosion resistance of coatings for use in turbo machinery, Wear, 186-187(Part 1) (1995) 224.

[16] Thapa B, Sand erosion in hydraulic machinery. Trondheim, Norwegian University of Science and Technology, Faculty of Engineering Science and Technology, Department of Energy and Process Engineering, 2004.

[17] Thapa B and Brekke H, Effect of sand particle size and surface curvature in erosion of hydraulic turbine, IAHR symposium on hydraulic machinery and systems, Stockholm, 2004.

[18] Zhao K, C Gu, F Shen, \& B Lou, Study on mechanism of combined action of abrasion and cavitation erosion on some engineering steels. Wear 162-164 (Part 2): 811-819, 1993. 